\title{
Inversión en programas en línea: caso de la maestría en Defensa del Contribuyente de la Universidad Michoacana de San Nicolás de Hidalgo en México
}

\begin{abstract}
Investment in online programs: the case for the Maestría en Defensa del Contribuyente at the Universidad Michoacana de San Nicolás de Hidalgo in Mexico
\end{abstract}

Investimento em programas online: caso do Mestrado em Defesa do Contribuinte da Universidade Michoacana de San Nicolás de Hidalgo, no

México

Priscila Ortega Gómez Universidad Michoacana de San Nicolás de Hidalgo, México

priscila.ortega@umich.mx https://orcid.org/0000-0002-3178-574X

Zoe Tamar Infante Jiménez Universidad Michoacana de San Nicolás de Hidalgo, México zoe.infante@umich.mx https://orcid.org/0000-0003-0223-2422

\section{Resumen}

La maestría en Defensa del Contribuyente es el primer programa de la Universidad Michoacana de San Nicolás de Hidalgo que se oferta a nivel nacional completamente en línea, administrando el uso de técnologías de la información de manera autónoma. En tal sentido, el objetivo de la presente investigación fue analizar a través de sus estudiantes el desempeño del programa para evaluar su alcance e identificar sus áreas de oportunidad para implementar medidas y estrategias de mejora. De igual manera, se pretendió mostrar evidencias de las ventajas y desafíos de este tipo de programas para que posteriormente puedan ser creados otros similares como una alternativa que requiere una reducida inversión. 


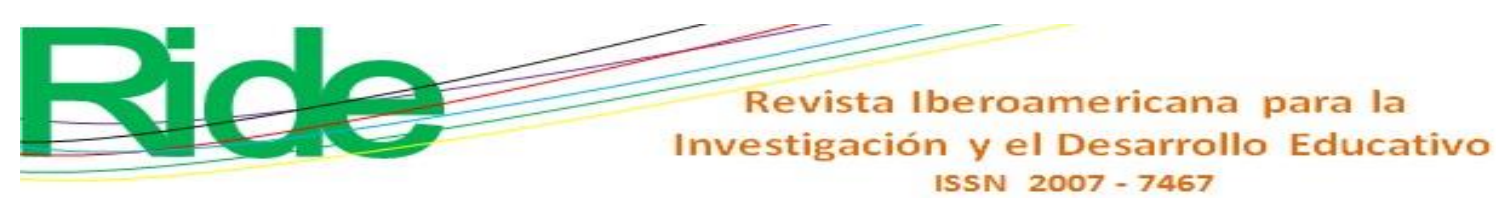

Para la recolección de la información se aplicó una encuesta de 50 preguntas en escala del 0 al 10 a los 34 estudiantes del programa de la primera y segunda generación mediante la plataforma Moodle; posteriormente, se procesaron y analizaron los datos mediante medidas de estadística descriptiva. Dentro de los principales resultados se detectó un nivel de satisfacción alto con el programa, principalmente porque les permite trabajar, superarse académicamente y actualizarse, así como porque pueden tomar sus sesiones desde su trabajo, hogar o cualquier lugar que tenga internet estable. Como áreas de oportunidad se detectó la necesidad de implementar acciones para que los estudiantes desarrollen en mayor medida las habilidades necesarias para aprovechar la transferencia de conocimiento mediante esta modalidad. Podemos concluir que el programa tiene grandes oportunidades de crecimiento, así como fortalezas y áreas de oportunidad, las cuales pueden ser atendidas para otorgar un servicio de mayor calidad. Si bien hay estudiantes que no están preparados para este sistema educativo, se considera que el programa les proporciona herramientas que contribuyen a desarrollar tales habilidades. Adicionalmente, durante estos meses de confinamiento debido a la pandemia, el programa no sufrió ninguna modificación ni contratiempo, a excepción de las cuestiones administrativas que son ajenas al control de este, lo que demuestra otras de sus ventajas.

Palabras clave: aprendizaje electrónico, educación a distancia, educación tecnológica, inversión en tecnología, tendencias del desarrollo educativo.

\section{Abstract}

Master's in Taxpayer Defense is the first program at the Universidad Michoacana de San Nicolás de Hidalgo that is offered nationwide completely online managing the use of information technologies in an autonomous manner. The objective of this research was to analyze through students the performance of the program to evaluate its scope, identifying areas of opportunity and thus be able to implement measures and strategies for improvement in the program. Likewise, it was intended to show evidence of the advantages and benefits of this type of program so that later on others can be created under this modality as an alternative that requires a reduced investment. For information gathering, a survey of 50 questions on a scale of zero to 10 was applied to the 34 students of the first- and secondgeneration program through the Moodle platform and later the data was processed and analyzed through descriptive statistical measures. Among the main results, a high level of 


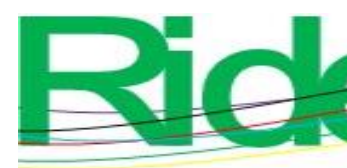

Revista Iberoamericana para la Investigación y el Desarrollo Educativo ISSN $2007-7467$

satisfaction with the online program was detected, mainly because it allows them to work, improve academically and update their skills, as well as because they can take their sessions from their work, home or any place that has a stable internet. As areas of opportunity, the need to implement actions was detected so that students could adapt and develop to a greater extent the skills needed to take advantage of the transfer of knowledge through this modality. We can conclude that the program has great opportunities for growth, has many strengths and the areas of opportunity detected are important tools to provide a higher quality program. It was detected that there are students who are not yet prepared for this educational system, but it is considered that the program provides them with tools that contribute to the development of such skills. Additionally, during these months of pandemic confinement, the program did not undergo any modifications or setbacks, except for administrative issues that are beyond the control of the program, which demonstrates some of the advantages and benefits of this type of program.

Keywords: electronic-learning, distance education, technology education, technology investment, educational development trends.

\section{Resumo}

O mestrado em Defesa do Contribuinte é o primeiro programa da Universidade Michoacana de San Nicolás de Hidalgo oferecido em todo o país de forma totalmente online, gerenciando o uso das tecnologias de informação de forma autônoma. Nesse sentido, o objetivo desta pesquisa foi analisar por meio de seus alunos o desempenho do programa para avaliar sua abrangência e identificar suas áreas de oportunidade para implementar medidas e estratégias de melhoria. Da mesma forma, pretendeu-se evidenciar as vantagens e desafios deste tipo de programa para que, posteriormente, outros semelhantes possam ser criados como alternativa que requeira um investimento reduzido. Para a coleta de informações, foi aplicado um levantamento de 50 questões em uma escala de 0 a 10 aos 34 alunos do programa de primeira e segunda geração utilizando a plataforma Moodle; posteriormente, os dados foram processados e analisados por meio de medidas estatísticas descritivas. Dentre os principais resultados, foi detectado um alto nível de satisfação com o programa, principalmente por permitir que trabalhem, se aprimorem academicamente e se atualizem, bem como por poderem realizar suas sessões no trabalho, em casa ou em qualquer lugar que tenha um Internet. Como áreas de oportunidade, detectou-se a necessidade de implementação de ações 


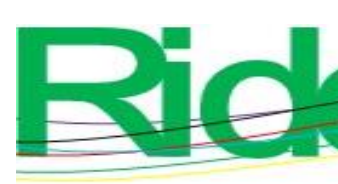

Revista Iberoamericana para la
Investigación y el Desarrollo Educativo
ISSN $2007-7467$

para que os alunos desenvolvam ainda mais as competências necessárias para o aproveitamento da transferência de conhecimentos por meio dessa modalidade. Podemos concluir que o programa apresenta grandes oportunidades de crescimento, bem como pontos fortes e áreas de oportunidade, que podem ser aproveitadas para prestar um serviço de maior qualidade. Embora existam alunos não preparados para este sistema de ensino, considera-se que o programa disponibiliza ferramentas que contribuem para o desenvolvimento dessas competências. Além disso, durante esses meses de confinamento devido à pandemia, o programa não sofreu qualquer modificação ou retrocesso, com exceção de questões administrativas que fogem ao seu controle, o que demonstra outras vantagens.

Palavras-chave: e-learning, educação a distância, educação tecnológica, investimento em tecnologia, tendências de desenvolvimento educacional.

Fecha Recepción: Octubre 2020

Fecha Aceptación: Mayo 2021

\section{Introducción}

La educación a distancia, a diferencia de la presencial, se puede concebir desde una perspectiva que engloba varias formas de enseñanza y aprendizaje que no están bajo la supervisión continua e inmediata de los tutores en una sala de conferencias o en las mismas instalaciones, pero que igualmente se benefician de la planificación, orientación y enseñanza de dichos profesionales (Kok, Bester y Esterhuizen, 2018).

Durante las últimas dos décadas, la educación a distancia ha pasado de la periferia al centro de la provisión de educación general. Este es especialmente el caso en el sector de la educación superior, donde en algunos países — con el apoyo de grandes programas de inversión y financiación estatal - han impulsado diversos programas de educación a distancia, mayormente conocidos como aprendizaje en línea, flexible o mixto. Cada año la matrícula de estudiantes en educación a distancia aumenta, así como el número de programas académicos ofertados. Entre los países que han experimentado un mayor crecimiento en las últimas décadas en la educación superior a distancia y en línea destacan Estados Unidos, China, India, Rusia, Australia, entre otros. La flexibilidad, el ahorro de tiempo y de costos ha generado este notable aumento, por lo que en la mayoría de los países desarrollados esta modalidad se ha convertido en una parte importante de la educación superior, ya que tiene la misma relevancia un título obtenido mediante un programa a distancia que uno presencial, aunque vale acotar que en otros países todavía es difícil lograr esto último (Zawacki y Qayyum, 2019). 


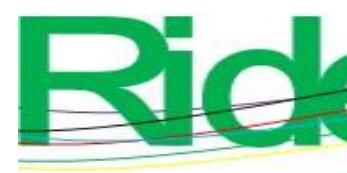

Revista Iberoamericana para la
Investigación y el Desarrollo Educativo
ISSN $2007-7467$

Por eso, se puede afirmar que la enseñanza superior ha experimentado diversas transformaciones, fundamentalmente en cuanto al auge de la internacionalización y de la movilidad estudiantil, la ampliación de las oportunidades de aprendizaje en línea y mixto, así como la proliferación de los servicios de internet. Estos cambios, sin embargo, demandan mejorar y garantizar la calidad y el reconocimiento de los programas, y exigen a los gobiernos e instituciones que implementen políticas innovadoras para incentivar la igualdad y la accesibilidad, así como el crecimiento de la educación (United Nations Educational, Scientific and Cultural Organitation [Unesco], 2019). De esta manera, las formas de enseñanza no presenciales han tenido un impacto favorable como alternativa formativa para grupos sociales que no pueden ajustarse a los ritmos de enseñanza escolarizada (Moreno y Cárdenas, 2012).

Desde la Declaración de París sobre los recursos educativos abiertos (REA) en el año 2012 (la cual recomienda a los Estados, en la medida de sus posibilidades y competencias, diez estrategias para promover la educación a distancia), solo un pequeño número de países ha desarrollado políticas nacionales para favorecer la educación a distancia y en línea. Aun así, el interés en esta modalidad con o sin implicaciones políticas ha crecido. En este sentido, los países están formulando diversos enfoques para implementar iniciativas que puedan conducir a abordar los desafíos educativos nacionales, desde aumentar el acceso hasta mejorar la calidad y reducir los costos en la educación. El enfoque del uso de estas modalidades se ha ampliado de la educación superior a otros niveles y sectores de la educación (Unesco, 2016).

Las diferentes naciones y sistemas educativos están respondiendo de manera diferente al macroproceso de digitalización. Algunos sistemas nacionales están más avanzados y trabajan en hacer que la digitalización de la enseñanza y el aprendizaje sea un objetivo estratégico para el desarrollo y la innovación (tal es el caso de Corea del Sur), mientras que en otros países la educación a distancia fue reconocida como una forma de provisión de educación validada y acreditada solamente. Actualmente, se registran altas tasas de crecimiento de matrícula en educación a distancia y en línea con instituciones privadas que ingresan masivamente a este mercado (p. ej., Brasil). No obstante, la transformación de la enseñanza y aprendizaje en una era digital presenta un desafío dramático de innovación y cambio para la mayoría de las universidades "convencionales".

Las instituciones de enseñanza a distancia siempre han encabezado la implementación de medios nuevos y emergentes, ya que en este sistema siempre se ha utilizado la tecnología 


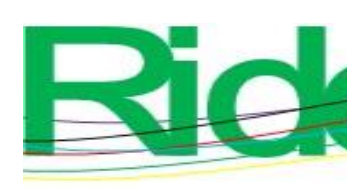

Revista Iberoamericana para la Investigación y el Desarrollo Educativo ISSN $2007-7467$

más reciente para cerrar la brecha entre los estudiantes y las instituciones de enseñanza, así como entre los propios estudiantes. A mediados de la década de 1990, el Internet y las nuevas tecnologías de información y comunicación abrieron el camino para superar la noción de educación a distancia como una forma aislada de aprendizaje (Zawacki y Qayyum, 2019).

Ciertamente, la evolución de la tecnología ha jugado un papel importante en la maduración del aprendizaje a distancia de una forma de educación "alternativa" a la corriente principal. La enseñanza a distancia es muy diferente de la instrucción tradicional en el aula, pues se necesitan diferentes habilidades de presentación y estrategias de enseñanza (Kearsley, 1998).

Para Bossu (2016) la apertura de las naciones ha contribuido a transformar la educación en todos los niveles. En la enseñanza superior se ha beneficiado a los alumnos, a los educadores, a las universidades, a los diseños institucionales y a las inversiones en investigación. Ha reunido a los dirigentes nacionales para debatir la forma en que las naciones más ricas podrían ayudar a las menos favorecidas a aumentar el acceso a la educación gratuita y abierta.

Durante la próxima década veremos una nueva generación de instructores que tomaron sus títulos a través de la educación a distancia, por lo que tendrán la experiencia vivencial para construir. Mucho más complicado es la cuestión del cambio institucional, dado que la educación a distancia requiere que las escuelas, las universidades y los departamentos de capacitación realicen cambios importantes en su forma de hacer las cosas. Además, casi todas las políticas y procedimientos que tienen que ver con el registro, la programación de clases, la calificación, la graduación y la asistencia probablemente tendrán que ser transformados para ajustarse a la educación a distancia (Kearsley, 1998).

Actualmente, el volumen de recursos educativos digitales impone nuevas exigencias a los sistemas e instituciones de la educación superior en cuanto al desarrollo de los programas educativos, los planes de estudio y los procesos de aprendizaje novedosos e innovadores, así como en las vías de acceso a la enseñanza superior. Todo ello ha sido propiciado por la existencia de modelos de prestación de servicios de aprendizaje en línea (educación abierta, mixta y cursos de corta duración) basados en la adquisición de competencias, tales como los cursos en línea masivos y de libre acceso (MOOC) y los recursos educativos abiertos (REA). El enorme potencial que propicia el aprendizaje en línea, en general, y la forma de MOOC, en particular, abre nuevas vías de acceso a la 


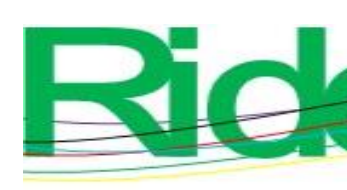

Revista Iberoamericana para la
Investigación y el Desarrollo Educativo
ISSN $2007-7467$

enseñanza superior, así como al aumento de las posibilidades de educación mediante soluciones alternativas flexibles (Unesco, 2019).

En este contexto, las generaciones más recientes se distinguen, principalmente, por el uso intensivo, extensivo y más accesible de tecnologías, lo que facilita los procesos de aprendizaje y su gestión. Este nuevo impulso se evidencia con el surgimiento no solo de programas o entidades de educación a distancia en entornos digitales dentro de las universidades tradicionalmente escolarizadas, sino también con la fundación de nuevas instituciones de educación superior públicas y privadas, lo que muestra un mayor dinamismo de estas últimas en la generación de programas académicos a distancia (Moreno, 2017).

De esta manera, con el advenimiento de Internet, la amenaza de la competencia de las nuevas organizaciones educativas virtuales ha obligado a las instituciones tradicionales a repensar cómo brindan instrucción y a desarrollar políticas y procedimientos más propicios para la educación a distancia, ya que en esta los límites geográficos no son pertinentes. Esto significa que cada escuela o universidad en el país y el mundo podría competir entre sí, lo que obligaría a las instituciones a pensar en la calidad y la singularidad de sus ofertas. En tal sentido, ahora la interacción virtual se puede lograr mediante una videoconferencia bidireccional, la cual puede reducir costos y ser más generalizada sobre todo para alumnos adultos que estudian a tiempo parcial para aprender según sus conveniencias, y no según las de su institución educativa. La educación a distancia que cumple este deseo es mucho más aceptable para ellos y, por lo tanto, exitosa. Es así como la educación a distancia satisface las necesidades de la civilización del siglo XXI (Kearsley, 1998).

Sin embargo, Kok et al. (2018) señalan que aunque los estudiantes pueden tener acceso a infraestructuras bien respaldadas y técnica de apoyo, no usan la tecnología a favor de la educación a menos que posean una actitud positiva hacia ella, la cual solo pueden conseguir desarrollando habilidades y aptitudes tecnológicas.

La educación superior en línea o a distancia ha beneficiado a los estudiantes y educadores e influido en la forma en que los altos ejecutivos de las universidades abordan los planes y políticas estratégicas institucionales. De hecho, ha sacudido los modelos empresariales universitarios establecidos e influido en el desarrollo de otros nuevos, y ha reunido a los líderes nacionales para discutir cómo los países más ricos podrían ayudar a los menos favorecidos a aumentar el acceso a la educación gratuita y abierta (Unesco, 2016).

Con base en lo anterior, el presente trabajo se centró en el estudio de caso de un programa académico de posgrado que se oferta completamente en línea en una universidad 


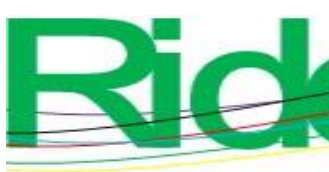

\section{Revista Iberoamericana para la Investigación y el Desarrollo Educativo ISSN 2007 - 7467}

mexicana. Para ello, primero se ofrece una contextualización general de la educación a distancia en México; posteriormente, se describe el programa académico de estudio y el método utilizado; después se presentan los resultados y la discusión, y finalmente se explican las conclusiones.

\section{La educación a distancia en México}

Bajo la influencia de la dinámica mundial, en Iberoamérica y en nuestro país se da inicio a la aparición de redes de colaboración entre los centros educativos del nivel superior, las dependencias gubernamentales y las organizaciones de la sociedad civil para renovar el modelo de relación existente entre ellos y para abrir canales de comunicación y cooperación que ampliarán las posibilidades para un incremento en la cobertura en la atención a la demanda (Coronado, 2017).

En México, la educación a distancia — también conocida como en línea, virtual o e-learning - ha reportado un amplio crecimiento en el ámbito de la educación superior a través de diversas formas y expresiones (Zubieta y Rama, 2015). Aun así, este sistema es todavía incipiente (Moreno, 2015), pues son principalmente las instituciones educativas privadas las que ofertan en mayor medida programas educativos en línea (tabla 1), aunque como señalan Moreno y Cárdenas (2012) el crecimiento no implica necesariamente calidad o adecuación.

Tabla 1. Especialidad, maestría y doctorado, sistema no escolarizado

\begin{tabular}{|l|r|r|r|r|}
\hline & \multicolumn{1}{|c|}{$\begin{array}{c}\text { Lugares } \\
\text { ofertados }\end{array}$} & \multicolumn{1}{c|}{$\begin{array}{c}\text { Matrícula } \\
\text { total }\end{array}$} & \multicolumn{1}{c|}{$\begin{array}{c}\text { Egresados } \\
\text { total }\end{array}$} & \multicolumn{1}{|c|}{$\begin{array}{c}\text { Graduados } \\
\text { total }\end{array}$} \\
\hline Instituciones públicas & 11126 & 20833 & 7591 & 4812 \\
\hline Intituciones privadas & 85659 & 91151 & 35373 & 21299 \\
\hline
\end{tabular}

Fuente: Elaboración propia con datos de la Anuies (ciclo escolar 2017-2018) (inicio de cursos)

Es hasta los años setenta cuando las universidades en México incursionan en la educación a distancia con influencia de las grandes universidades europeas que adoptaban esta modalidad. La Universidad Autónoma de México (UNAM) fue la pionera en la implementación y crecimiento de estos programas.

Esta modalidad ha crecido tanto en nuestro país que actualmente la matrícula registrada en posgrado en sistema no escolarizados representa $47 \%$ de la matrícula en 


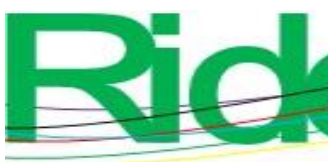

Revista Iberoamericana para la Investigación y el Desarrollo Educativo ISSN $2007-7467$

sistema escolarizado. En la tabla 2 podemos observar la actual importancia de esta modalidad en la educación en posgrado.

Tabla 2. Posgrado por sistema: especialidad, maestría y doctorado

\begin{tabular}{|l|r|r|r|r|}
\hline & \multicolumn{1}{|c|}{$\begin{array}{c}\text { Lugares } \\
\text { ofertados }\end{array}$} & Matrícula total & $\begin{array}{c}\text { Egresados } \\
\text { total }\end{array}$ & $\begin{array}{c}\text { Graduados } \\
\text { total }\end{array}$ \\
\hline Escolarizados & 135991 & 239948 & 81635 & 66016 \\
\hline No escolarizados & 96785 & 111984 & 42964 & 26111 \\
\hline
\end{tabular}

Fuente: Elaboración propia con datos de la Anuies (ciclo escolar 2017-2018) (inicio de cursos)

De acuerdo con la página Universidades virtuales en México, actualmente se ofertan más de 2300 licenciaturas, maestrías y cursos en la modalidad en línea y a distancia, con una lista de las 25 universidad públicas y 32 privadas con diversos programas en estas modalidades, aunque se cree que hay muchas más privadas. En el ranking de las mejores universidades en línea, la UNAM se ecuentra colocada en la primera posición, seguida de la Universidad Abierta y a Distancia de México (UNADM) y la Universidad Virtual del Estado de Guanajuato (UVEG), por mencionar algunas de las mejor puntuadas (Licenciaturas en línea, 2019).

En México, 37 \% de los estudiantes que reciben educación en línea tienen entre 25 y 29 años de edad, $27 \%$ entre 18 y 24 años, $19 \%$ entre 30 y 34 años, y $44 \% 35$ años o más. Solamente $1 \%$ tiene menos de 18 años. Asimismo, $40 \%$ estudian una licenciatura o ingeniería y solo $7 \%$ estudia una maestría. Al hablar exclusivamente de quienes actualmente estudian en línea, la flexibilidad de horarios es el principal aspecto considerado para decidirse por esta modalidad (Asociación de internet MX y OCC Mundial, 2018).

Por otra parte, $39 \%$ de la matrícula en posgrado o especialidad en sistema no escolarizado tienen entre 24 y 29 años y $33 \%$ entre 30 y 39 años (tabla 3).

Tabla 3. Matrícula por especialidad, maestría y doctorado en sistema no escolarizado Matrícula menores de 23 años 8623 


\begin{tabular}{|l|r|}
\hline Matrícula 24 a 29 años & $\begin{array}{c}\text { Revista Iberoamericana para la } \\
\text { Investigación y el Desarrollo Educativo } \\
\text { ISsN } \\
2007-7467\end{array}$ \\
\hline Matrícula 30 a 39 años & 36525 \\
\hline Matrícula 40 años y mayores & 23567 \\
\hline
\end{tabular}

Fuente: Elaboración propia con datos de la Anuies (ciclo escolar 2017-2018) (inicio de

cursos)

Históricamente, hay una preocupación constante sobre la necesidad de llegar a acuerdos para el trabajo y el aval interinstitucional, ya sea por parte de las instituciones de educación superior (IES) o del Gobierno, cuyos esfuerzos han tenidos altibajos y no han llegado a consolidarse en gran medida debido a la falta de acuerdos reales — no solo documentales - entre algunas instancias; por ejemplo, entre la Asociación Nacional de Universidades e Instituciones de Educación Superior (Anuies) y la Secretaría de Educación Pública (SEP), acuerdos que sí se han alcanzado entre las instituciones de educación superior (Moreno, 2015).

De manera general, podemos decir que la educación a distancia es una mezcla de tradición y modernidad que se desarrolla en una pugna entre la rutina y la innovación, pues por una parte se propician nuevas condiciones académicas y, por otra, perduran las prácticas burocráticas escolares. Sin duda, se registran cambios tecnológicos en la investigación que toma estas modalidades educativas como objeto de estudio, lo mismo que en el posicionamiento social y en el reconocimiento internacional; pero los cambios son menores en las políticas, la organización y los estilos de gestión administrativa (Moreno, 2015). Adicionalmente, Moreno (2015) señala la existencia de amplios sectores de la población que, por sus condiciones tanto socioeconómicas como académicas y laborales, se vuelven aspirantes potenciales para el ingreso a sistemas e-learníng; sin embargo, sus posibilidades de adaptación al sistema se ven mermadas por su escaso dominio en el uso de recursos tecnológicos y, sobre todo, por causa de los déficits existentes en términos de habilidades autorregulatorias adquiridas. En contraste, Espinosa (2017) señala que no es muy difícil caracterizar el escenario actual, donde probablemente haya estudiantes "enganchados", capaces de conectarse y acceder con gran habilidad a los conocimientos "más actualizados" con rapidez y profesores "desconectados", "a la antigua", lo que genera un desequilibrio en las relaciones entre ambos y la posible falacia de desplazamiento hacia una preponderancia de los estudiantes en sus vínculos con los profesores. 


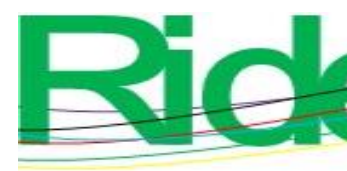

Revista Iberoamericana para la
Investigación y el Desarrollo Educativo
ISSN $2007-7467$

El desarrollo acelerado de la sociedad de la información ha planteado retos para la educación y el aprendizaje. En tal sentido, la incorporación de las tecnologías de la información en la educación es una pieza clave para llevar a cabo las transformaciones necesarias en los programas en línea y a distancia, así como la integración, capacitación, actualización y adecuación de los docentes y la implementación de estrategias para la transferencia y adquisición de conocimiento por parte de los estudiantes, los cuales deben desarrollar conductas autodidactas.

\section{La maestría en Defensa del Contribuyente}

La maestría en Defensa del Contribuyente (MDC) es un programa de reciente creación, aprobado por el H. Consejo Universitario de la UMSNH en agosto de 2016. La primera convocatoria fue emitida a finales del año 2016 y el inicio del primer semestre de la primera generación se produjo en agosto de 2017.

Es el primer programa de la UMSNH que se oferta a nivel nacional completamente en línea, administrando el uso de TIC de manera autónoma. En julio de 2019 egresó la primera generación, aunque su titulación fue compleja debido a que había estudiantes de diversos estados de la República y solo existe el mecanismo tradicional para llevar a cabo los trámites administrativos. El programa es semestral y se divide en dos trimestres: en el primero se cursan dos materias y en el segundo otras dos. A partir del semestre 2019-2020, para mayor aprovechamiento, se implementó el cursar una materia a la vez de manera intensiva.

Las TIC que comenzó utilizando la maestría fueron la plataforma de Moodle y las videoconferencias en Adobe Connect. Actualmente, aprovechando los recursos que se han puesto a disposición de manera gratuita por parte de la universidad, se ha sustituido Adobe Connect por Meet de Google, con lo cual se redujo el gasto anual de esta herramienta. Además, se capacitan a los profesores y se elaboran tutoriales para que los estudiantes aprendan a usar dichos recursos.

Debido al creciente interés por parte de personas de diversas edades en cursar la maestría, se puede inferir que el programa está desempeñándose de manera adecuada; sin embargo, surgen interrogantes como las siguientes: ¿en qué medida han funcionado las herramientas y recursos tecnológicos que se han invertido para implementar la maestría en Defensa del Contribuyente y cuál ha sido el nivel de aceptación de los estudiantes ante el uso 

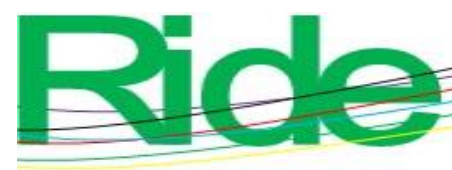

Revista Iberoamericana para la Investigación y el Desarrollo Educativo

ISSN $2007-7467$

de estas? ¿Hasta qué punto es factible la creación de otros programas en la universidad que utilicen los mismos recursos para su funcionamiento?

Por ello, en la presente investigación se procuró analizar, a través de la opinión de los estudiantes, el desempeño del programa de la maestría en Defensa del Contribuyente con la finalidad de evaluar su alcance e identificar las áreas de oportunidad para implementar medidas y estrategias de mejoras. Para ello, se enseñan evidencias de las ventajas y beneficios de este tipo de programas para futuras iniciativas similares.

\section{Método}

Para cumplir con el objetivo establecido, la evaluación se efectuó mediante la medición y análisis de la percepción de los estudiantes sobre el desempeño del programa y su forma de aprovechamiento. Esto permitió identificar áreas de oportunidad que podrán servir para elaborar estrategias que contribuyan a su mejora.

\section{Datos}

Se aplicó una encuesta de 50 preguntas a los 34 estudiantes de la primera y la segunda generación mediante la plataforma de Moodle.

Se utilizó una escala de 0 a 10 a los estudiantes en relación con la percepción que tienen respecto a diversas variables relacionadas con el programa de la maestría. La elección de variables se basó en una revisión de la literatura que sirvió para seleccionar las siguientes (tabla 4):

Tabla 4. Variables y número de ítems considerados en la encuenta 


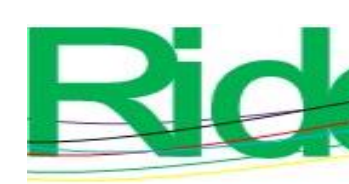

Revista Iberoamericana para la Investigación y el Desarrollo Educativo ISSN 2007 - 7467

\begin{tabular}{|l|c|}
\hline Variables & $\begin{array}{c}\text { N. }{ }^{\circ} \text { de } \\
\text { ítems }\end{array}$ \\
\hline Organización & 3 \\
\hline Estructura curricular & 1 \\
\hline Mecanismos de operación y recursos tecnológicos invertidos & 5 \\
\hline Interactividad & 8 \\
\hline Accesibilidad & 9 \\
\hline Flexibilidad de tiempo & 6 \\
\hline Horarios de videoconferencias & 2 \\
\hline Incorporación de recursos & 4 \\
\hline Colaborativa & 3 \\
\hline Comunicación & 9 \\
\hline
\end{tabular}

Fuente: Elaboración propia

A continuación, se ofrece una breve explicación del significado de cada variable:

- Organización: Qué tan adecuada resulta la planeación y estructura del programa educativo.

- Estructura curricular: Qué tan atractivo y/o adecuado resulta el plan de estudios del programa educativo.

- Mecanismos de operación y recursos tecnológicos: Qué tan adecuados y eficientes resultan los recursos tecnológicos utilizados y los mecanismos de operación en el programa educativo.

- Interactividad: Qué tanto el estudiante puede conectarse de manera más dinámica con sus compañeros, docentes y con el material educativo.

- Accesibilidad: Qué tanto el estudiante puede acceder a sus cursos de educación en línea desde el lugar en el que se encuentre, en cualquier momento del día.

- Flexibilidad de tiempo: Qué tanta flexibilidad existe en el plan de estudios en relación con las videoconferencias y qué tanta en las actividades que se realizan sin esta sincronía.

- Incorporación de recursos: Qué tan útiles son los recursos utilizados, tanto tecnológicos como metodológicos, según los requerimientos de la sociedad y el entorno cambiante.

- Colaborativa: Qué tanta interacción y contacto se tiene entre los estudiantes (tales como labores de investigación colaborativas entre estudiantes) y estudiante-profesor utilizando para ello recursos como los chats o foros de discusión.

- Comunicación: Qué tanto facilitan la comunicación las TIC utilizadas en el programa (Enfoque estratégico, 2019). 

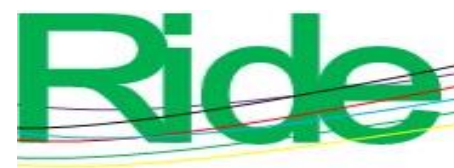

Revista Iberoamericana para la Investigación y el Desarrollo Educativo ISSN 2007 - 7467

Una vez contestadas las encuentas, se procesaron y analizaron los datos mediante medidas de estadística descriptiva.

\section{Resultados}

En la figura 1 podemos observar la calificación que los estudiantes le otorgaron al programa de la MDC de manera general. Los resultados señalan que $65 \%$ de los estudiantes le otorgan una calificación entre muy buena y excelente.

Figura 1. Calificación otorgada al programa de la maestría en Defensa del Contribuyente

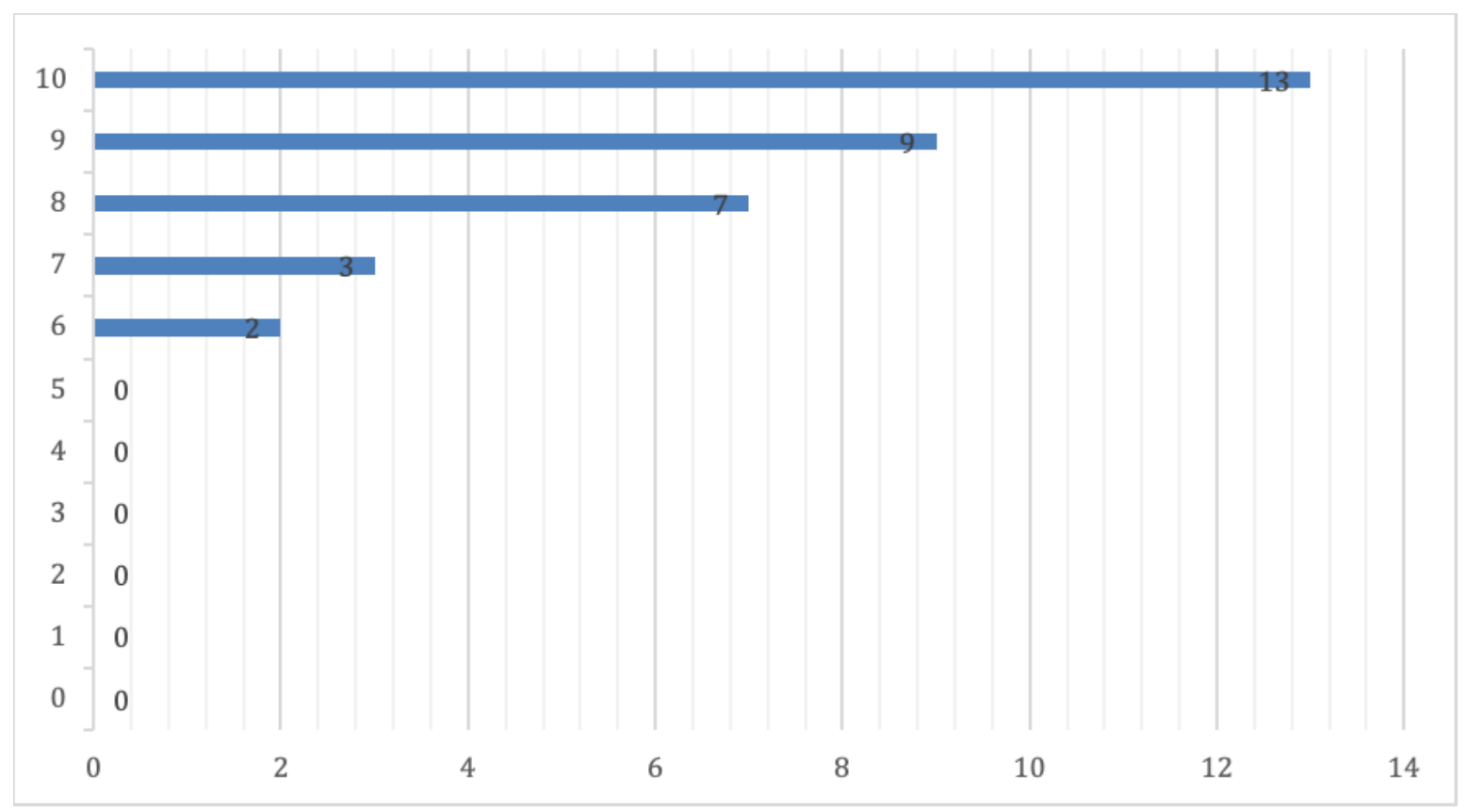

Fuente: Elaboración propia

Respecto a la variable organización, el promedio general otorgado al programa fue 8.8. Asimismo, la calificación 10 fue la que más veces se repitió, con una mediana de 9.

En tabla 5 se presentan los principales resultados de las medidas estadísticas de tendencia central. 


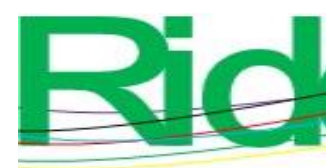

Tabla 5. Resumen de resultados de encuesta de percepción a estudiantes de la MDC

\begin{tabular}{|c|c|c|c|}
\hline Preguntas & Promedio & Moda & Mediana \\
\hline $\begin{array}{l}\text { 1. En general, ¿qué calificación le otorgarías al programa } \\
\text { de la maestría? }\end{array}$ & 8.8 & 10 & 9 \\
\hline $\begin{array}{l}\text { 2. ¿Consideras adecuado que la planeación del programa } \\
\text { sea semestral? }\end{array}$ & 9.1 & 10 & 9.5 \\
\hline $\begin{array}{l}\text { 3. ¿Consideras adecuado que el semestre se divida en dos } \\
\text { partes: en la primera para cursar dos materias y en la } \\
\text { segunda otras dos? }\end{array}$ & 9.6 & 10 & 10 \\
\hline $\begin{array}{l}\text { 4. ¿Consideras adecuadas las materias que se imparten en } \\
\text { el programa? }\end{array}$ & 9.1 & 10 & 9 \\
\hline $\begin{array}{l}\text { 5. ¿Qué calificación le otorgarías a la página de la } \\
\text { maestría? }\end{array}$ & 9.3 & 10 & 9 \\
\hline $\begin{array}{l}\text { 6. ¿Qué tan sencillo te resulta encontrar la información que } \\
\text { buscas en la página de la maestría? }\end{array}$ & 9.3 & 10 & 9 \\
\hline $\begin{array}{l}\text { 7. ¿Qué calificación le otorgas a la plataforma de Moodle } \\
\text { de la MDC? }\end{array}$ & 9.2 & 10 & 9 \\
\hline $\begin{array}{l}\text { 8. ¿Qué calificación le otorgas a la Adobe Connect para las } \\
\text { videoconferencias? }\end{array}$ & 9.0 & 10 & 9 \\
\hline $\begin{array}{l}\text { 9. Considerando que el sistema de educación en línea es en } \\
\text { su mayoría autodidacta, ¿qué tan adecuados crees que son } \\
\text { los recursos didáctico-metodológicos en esta maestría? }\end{array}$ & 8.9 & 10 & 9 \\
\hline $\begin{array}{l}\text { 10. ¿Qué tan fácil te ha resultado utilizar la plataforma de } \\
\text { Moodle para revisar las actividades, tareas, } \\
\text { investigaciones, etc.? }\end{array}$ & 9.1 & 10 & 9 \\
\hline $\begin{array}{l}\text { 11. ¿Qué tan fácil te ha resultado utilizar la plataforma de } \\
\text { Moodle para subir las actividades, tareas, investigaciones, } \\
\text { etc.? }\end{array}$ & 9.3 & 10 & 9 \\
\hline $\begin{array}{l}\text { 12. ¿Qué tan fácil te ha resultado utilizar la plataforma de } \\
\text { Moodle para comunicarte con tus maestros? }\end{array}$ & 8.7 & 9 & 9 \\
\hline $\begin{array}{l}\text { 13. ¿Qué tan fácil te ha resultado utilizar la plataforma de } \\
\text { Moodle para comunicarte con tus compañeros? }\end{array}$ & 8.5 & 9 & 9 \\
\hline $\begin{array}{l}\text { 14. ¿Qué tan fácil te ha resultado utilizar las la plataforma } \\
\text { Adobe Connect de videoconferencias semanales para } \\
\text { comunicarte con tus maestros? }\end{array}$ & 9.0 & 10 & 9 \\
\hline $\begin{array}{l}\text { 15. ¿Qué tan fácil te ha resultado utilizar la plataforma } \\
\text { Adobe Connect de videoconferencias semanales para } \\
\text { comunicarte con tus compañeros? }\end{array}$ & 8.9 & 9 & 9 \\
\hline $\begin{array}{l}\text { 16. ¿Qué tan fácil te ha resultado utilizar la plataforma } \\
\text { Adobe Connect de videoconferencias semanales para } \\
\text { aclarar dudas con tus maestros? }\end{array}$ & 8.8 & 9 & 9 \\
\hline $\begin{array}{l}\text { 17. ¿Qué tan fácil te ha resultado utilizar la plataforma } \\
\text { Adobe Connect de videoconferencias semanales para } \\
\text { efectuar alguna solicitud? }\end{array}$ & 8.6 & 9 & 9 \\
\hline $\begin{array}{l}\text { 18. ¿Qué tan fácil te resulta ingresar a la plataforma de } \\
\text { Moodle para poder realizar tus tareas y actividades } \\
\text { semanales? }\end{array}$ & 9.2 & 10 & 9.5 \\
\hline
\end{tabular}




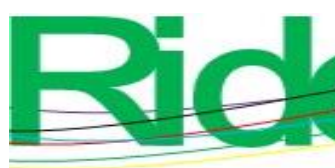

Revista Iberoamericana para la Investigación y el Desarrollo Educativo ISSN 2007 - 7467

19. ¿Qué tan común es que ingreses a la plataforma de Moodle de la MDC desde tu trabajo?

20. ¿Qué tan común es que ingreses a la plataforma de Moodle de la MDC desde tu casa?

21. ¿Qué tan común es que ingreses a la plataforma de Moodle de la MDC desde otros sitios (central de autobuses, aeropuerto, un evento, etc.)?

22. ¿Qué tan fácil te resulta ingresar a la plataforma Adobe Connect de videoconferencias semanales?

23. ¿Qué tan común es que ingreses a la plataforma de Adobe Connect de videoconferencias semanales desde tu trabajo?

24. ¿Qué tan común es que ingreses a la plataforma de Adobe Connect de videoconferencias semanales desde tu casa?

25. ¿Qué tan común es que ingreses a la plataforma de Adobe Connect de videoconferencias semanales desde un café o restaurante?

26. ¿Qué tan común es que ingreses a la plataforma de Adobe Connect de videoconferencias semanales desde otros sitios (central de autobuses, aeropuerto, un evento, etc.)?

27. ¿Lo más común es que realices tus tareas y actividades en horario matutino entre semana?

28. ¿Lo más común es que realices tus tareas y actividades en horario vespertino entre semana?

29. ¿Lo más común es que realices tus tareas y actividades en horario nocturno entre semana?

30. ¿Lo más común es que realices tus tareas y actividades durante los fines de semana?

31. ¿Te resulta adecuado el tiempo que dispones para efectuar las actividades semanales?

32. ¿Qué tan accesible te ha resultado que la planeación de las actividades sean semanales?

33. ¿Qué tan accesibles te han resultado los horarios establecidos para las videoconferencias?

34. ¿Qué tan probable es que un maestro acceda a cambiar el horario de la videoconferencia cuando lo solicita el grupo?

35. ¿En qué medida consideras adecuados los recursos utilizados por los maestros para transferirte conocimiento? 36. ¿En qué medida consideras adecuados los recursos utilizados por los maestros para consultar material y bibliografía? 37. ¿En qué medida consideras adecuados los recursos utilizados por los maestros para motivarte al aprendizaje autodidacta?

\begin{tabular}{|c|c|c|}
\hline 8.9 & 10 & 10 \\
\hline 8.7 & 10 & 9 \\
\hline 6.2 & 8 & 7.5 \\
\hline 9.0 & 9 & 9 \\
\hline 8.4 & 10 & 9 \\
\hline 8.0 & 10 & 9 \\
\hline 5.9 & 8 & 7.5 \\
\hline 5.4 & 0 & 7 \\
\hline 4.8 & 0 & 6.5 \\
\hline 7.1 & 9 & 8 \\
\hline 9.3 & 10 & 10 \\
\hline 8.9 & 10 & 10 \\
\hline 7.0 & 9 & 8 \\
\hline 7.8 & 10 & 8.5 \\
\hline 7.9 & 9 & 9 \\
\hline 8.6 & 10 & 9 \\
\hline 8.6 & 9 & 9 \\
\hline 8.7 & 9 & 9 \\
\hline 8.6 & 9 & 9 \\
\hline
\end{tabular}




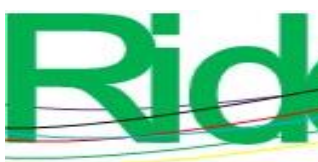

Revista Iberoamericana para la Investigación y el Desarrollo Educativo ISSN 2007 - 7467

\begin{tabular}{|c|c|c|c|}
\hline $\begin{array}{l}\text { 38. ¿En qué medida consideras adecuados los recursos } \\
\text { utilizados por los maestros para estimular la investigación? }\end{array}$ & 8.4 & 8 & 8.5 \\
\hline $\begin{array}{l}\text { 39. ¿Qué tan frecuente es que los profesores organicen } \\
\text { actividades en las que tengas que trabajar en conjunto con } \\
\text { algunos de tus compañeros? }\end{array}$ & 7.1 & 8 & 8 \\
\hline $\begin{array}{l}\text { 40. ¿Qué tan frecuente es que consultes a tus compañeros } \\
\text { para aclarar alguna duda? }\end{array}$ & 8.2 & 10 & 9 \\
\hline $\begin{array}{l}\text { 41. ¿Qué tan frecuente es que te organices con algunos de } \\
\text { tus compañeros para desarrollar alguna actividad aunque } \\
\text { no lo haya solictado de esa manera el profesor? }\end{array}$ & 6.6 & 9 & 8 \\
\hline $\begin{array}{l}\text { 42. ¿Cómo calificarías la comunicación que brinda la } \\
\text { plataforma de Moodle entre profesor-alumno? }\end{array}$ & 8.2 & 9 & 8.5 \\
\hline $\begin{array}{l}\text { 43. ¿Cómo calificarías la comunicación que brinda la } \\
\text { plataforma de Moodle entre alumno-alumno? }\end{array}$ & 7.8 & 9 & 8 \\
\hline $\begin{array}{l}\text { 44. ¿Qué tan útil te resulta que se publiquen los } \\
\text { comunicados en la pestaña de avisos de la página de la } \\
\text { maestría? }\end{array}$ & 8.9 & 10 & 9 \\
\hline $\begin{array}{l}\text { 45. ¿Qué tan útil te resulta que te envíen correo de los } \\
\text { comunicados de la maestría? }\end{array}$ & 9.8 & 10 & 10 \\
\hline $\begin{array}{l}\text { 46. ¿Cuando necesitas comunicarte con la coordinación de } \\
\text { la maestría es más común que lo hagas por correo } \\
\text { electrónico? }\end{array}$ & 8.7 & 10 & 10 \\
\hline $\begin{array}{l}\text { 47. ¿Cuando necesitas comunicarte con la coordinación de } \\
\text { la maestría es más común que lo hagas por teléfono? }\end{array}$ & 6.0 & 10 & 6 \\
\hline $\begin{array}{l}\text { 48. ¿Cuando necesitas comunicarte con la coordinación de } \\
\text { la maestría es más común que lo hagas por la plataforma } \\
\text { de Moodle? }\end{array}$ & 6.2 & 8 & 8 \\
\hline $\begin{array}{l}\text { 49. ¿Cuando necesitas comunicarte con la coordinación de } \\
\text { la maestría es más común que lo hagas por Whatsapp? }\end{array}$ & 5.9 & 10 & 7.5 \\
\hline $\begin{array}{l}\text { 50. ¿Cuando necesitas comunicarte con la coordinación de } \\
\text { la maestría es más común que lo hagas físicamente en la } \\
\text { coordinación? }\end{array}$ & 3.8 & 0 & 2.5 \\
\hline
\end{tabular}

Fuente: Elaboración propia

Los estudiantes consideran muy bueno el programa de la MDC. La mayoría cree que es muy adecuado que el semestre se divida en dos partes, cursando en la primera parte dos materias y otras dos en la segunda parte.

En cuanto a la estructura, perciben como adecuadas las materias que se imparten y piensan que la página de la maestría es muy buena, ya que es sencillo encontrar la información que buscan.

En relación con los mecanismos de operación y recursos tecnológicos que utiliza el programa para impartir las materias, así como la interactividad, creen que la plataforma de Moodle es muy buena, al igual que el programa de Adobe Connect para realizar las 


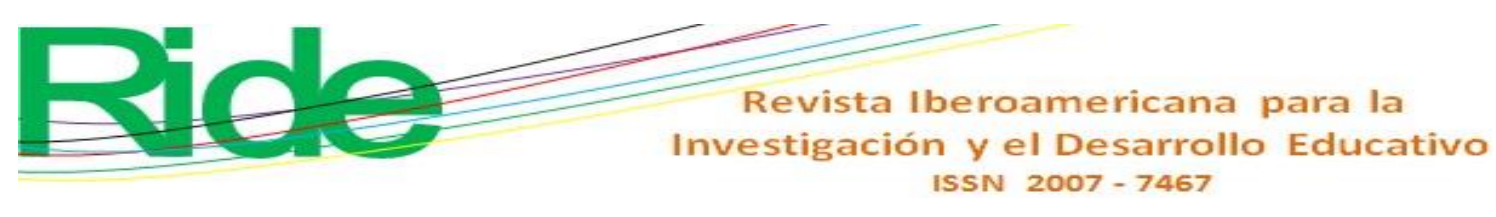

videoconferencias, ya que es accesible para revisar tareas, subirlas, aclarar dudas, comunicarse con los maestros y compañeros, y efectuar alguna solicitud.

Asimismo, consideran adecuados los recursos didáctico-metodológicos, aunque se ha detectado un déficit en varios estudiantes para ser autodidactas.

Referente a la accesibilidad, lo más común es que ingresen a la plataforma de Moodle y a las videoconferencias semanales desde su trabajo, aunque también lo hacen (pero menos frecuentemente) desde su casa, e incluso menos desde otros lugares como la central de autobuses, el aeropuerto, un evento, etc.

Concerniente a la flexibilidad de los tiempos, sabiendo del lapso que disponen (una semana) para entregar trabajos y tareas, lo más común es que los realicen en horario nocturno entre semana, seguido de los fines de semana y con menor frecuencia en horario vespertino entre semana, y aun menos en horario matutino entre semana.

Sobre el tiempo que disponen para efectuar las actividades semanales, lo consideran regular, lo que denota cierta falta de planeación y organización. En cuanto a la accesibilidad para los horarios de las videoconferencias, el promedio es bueno, ya que regularmente se imparten los jueves o los viernes después de las 6:00 p. m. Asimismo, cuando la mayoría de los estudiantes tiene problemas para asistir, el profesor acuerda un cambio de hora y/o día.

En relación con la incorporación de los recursos usados por los maestros para transferir el conocimiento, para consultar material y bibliografía, para motivar al aprendizaje autodidacta y para estimular la investigación, son considerados como buenos y muy buenos.

Sobre las actividades colaborativas, opinan que los profesores las estimulan de manera regular. Sin embargo (según el testimonio que han brindado de manera particular algunos estudiantes), en algunas ocasiones en los trabajos en equipo solo una persona realiza todo el trabajo, por lo que creen que no es una estrategia adecuada.

En cuanto a la comunicación, se ha detectado una muy buena comunicación entre el grupo, ya sea para resolver dudas de las tareas, dudas generales o para ponerse de acuerdo en alguna propuesta o solicitud. En tal sentido, Whatsapp es el medio más utilizado, mientras que para la comunicación con los profesores les parecen más adecuadas la plataforma de Moodle y las videoconferencias.

Finalmente, les resulta más útil que los comunicados de la coordinación de la maestría sean enviados por correo electrónico. De hecho, este recurso les parece ser el más idóneo para contactarse con dicha instancia, y en menor medida por Whatsapp o de forma personal. 


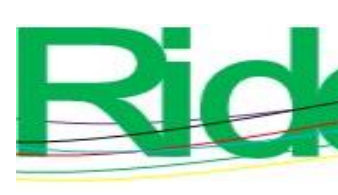

Revista Iberoamericana para la
Investigación y el Desarrollo Educativo
ISSN $2007-7467$

\section{Discusión}

Con base en la información recabada, se puede afirmar que se identificó un buen desempeño del programa de la MDC, con un nivel de satisfacción de los estudiantes que osciló entre bueno y muy bueno; sin embargo, también se detectaron algunas áreas de oportunidad, tales como establecer estrategias para contribuir al desarrollo de las habilidades autodidactas de los alumnos.

En cuanto a la organización, si bien le otorgaron una muy buena calificación — pues indicaron que el tiempo que disponen para la entrega de trabajos y tareas y para asistir a las videoconferencias es entre regular y bueno-, se ha propuesto una nueva estrategia en cuanto a la planeación de los cursos. En este sentido, a partir del semestre 2019-2020 los estudiantes cursarán una materia a la vez de manera intensiva, ya que se considera que de esta manera será más fácil centrar esfuerzos en una sola asignatura.

Para una mayor flexibilidad en relación con las videoconferencias, estas son grabadas para que posteriormente las puedan ver quienes no hayan podido asistir o para reforzar conocimientos.

En relación con la herramienta que se utiliza para las videoconferencias, se considera que Adobe Connect puede mejorarse o cambiarse por otra menos costosa y más amigable con los equipos de cómputo.

En cuanto a la comunicación estudiante-coordinación de la MDC (aun cuando se utilizan la plataforma Moodle, la videoconferencia y los chats, así como el correo electrónico), algunos estudiantes todavía optan por el teléfono. Esto evidencia la falta de adecuación al uso de las tecnologías más utilizadas en la modalidad virtual; sin embargo, también debemos tener presente que el rango de edad de los estudiantes de la maestría es muy amplio, pues abarca desde los 27 años hasta más de 40 años, siendo estos últimos a los que les ha costado un poco más acostumbrarse a los recursos tecnológicos implementados.

Referente a los materiales didácticos y tecnológicos, en cada semestre se lleva a cabo una reunión de capacitación con el objetivo de continuar mejorando, detectar fallas y buscar soluciones.

Finalmente, en cuanto a los procesos administrativos en todas las etapas (desde la inscripción hasta la titulación), la UMSNH los lleva a cabo de manera tradicional, es decir, el estudiante debe acudir de manera personal para entregar documentos originales que luego son digitalizados. Asimismo, para la entrega de credenciales y para la revisión del expediente 


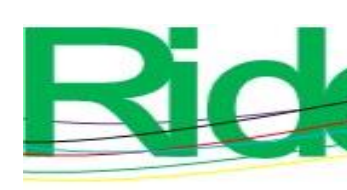

Revista Iberoamericana para la
Investigación y el Desarrollo Educativo
ISSN $2007-7467$

el interesado debe acudir, lo cual constituye un obstáculo. Por eso, la UMSNH debe trabajar en implementar estrategias administrativas para facilitar ese tipo de trámites.

En síntesis, es un hecho que actualmente la sociedad demanda una mayor diversificación de la educación para poder acceder a ella. En tal sentido, la UMSNH debe integrarse a este sistema no para erradicar la educación presencial, sino para tener la capacidad de ofrecer programas a los cuales puedan optar más personas, lo cual demanda modificaciones sustanciales tanto en cuestiones administrativas como académicas.

\section{Conclusiones}

A partir de los resultados enseñados en este trabajo, se puede concluir que los estudiantes consideran que el programa de la MDC tiene un buen desempeño, con un nivel de satisfacción que oscila entre bueno y muy bueno.

Asimismo, se puede indicar que la falta de adecuación al uso de las tecnologías más utilizadas en esta modalidad se presenta entre los estudiantes de mayor edad, por lo que como área de oportunidad - se debe mejorar la comunicación vía telefónica y se debe pensar en la elaboración de videos que expliquen detalladamente el uso y aplicación de las herramientas.

Además, se ha detectado que las bajas de estudiantes en el programa se relacionan con la falta de capacidades y/o actitudes para cursar un programa en línea, es decir, falta de organización del tiempo disponible, escasa preparación para recibir una educación autodidacta, ausencia del hábito lector, etc. Para atender estas carencias, sin embargo, se pueden mejorar las herramientas didácticas, lo cual también implica una mayor preparación de los docentes.

Por otra parte, se debe tener en cuenta que la conexión a internet en nuestro país (herramienta esencial para este tipo de programas) presenta muchas fallas, lo que puede representar un obstáculo difícil de sortear.

Aun así, podemos concluir que el programa analizado tiene grandes oportunidades de crecimiento, con fortalezas y áreas de oportunidad que deben seguir siendo evaluadas para procurar ofrecer el mejor servicio posible. En este sentido, no podemos negar que la sociedad demanda una mayor diversificación de la educación, lo cual se puede conseguir con la formación en línea. Al respecto, téngase en cuenta que esta es un metodología que pudiera servir para incrementar la matrícula con una inversión monetaria razonable, aunque para ello 


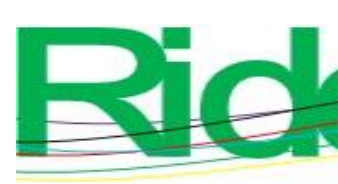

Revista Iberoamericana para la
Investigación y el Desarrollo Educativo
ISSN $2007-7467$

se necesitan modificaciones sustanciales tanto en cuestiones administrativas como académicas.

\section{Referencias}

Asociación de internet MX y OCC Mundial (2018). Estudio sobre educación en línea 2017. Recuperado de https://www.infotec.mx/es_mx/infotec/educacion_en_linea_en_mexico_2017

Asociación Nacional de Universidades e Instituciones de Educación Superior [Anuies] (2019). Anuario estadístico: población escolar en la educación superior. Posgrado. Ciclo escolar 2017-2018. Recuperado de www.anuies.mx-anuario-estadistico-deeducacion-superior

Bossu C. (2016). Open educational practices in Australia. In Miao, F., Mishra, S. and McGreal R. (eds.), Open educational resources: policy, costs, transformation (pp. 13-26). $\quad$ Retrieved from https://unesdoc.unesco.org/in/documentViewer.xhtml?v=2.1.196\&id=p::usmarcdef_ 0000244365\&file=/in/rest/annotationSVC/DownloadWatermarkedAttachment/attac h_import_e2785a23-68a7-4e0d-a086bc1e451601e3\%3F_\%3D244365eng.pdf\&locale=es\&multi=true\&ark=/ark:/48223/ pf0000244365/PDF/244365eng.pdf\#\%5B\%7B\%22num\%22\%3A120\%2C\%22gen\% 22\%3A0\%7D\%2C\%7B\%22name\%22\%3A\%22XYZ\%22\%7D\%2Cnull\%2Cnull\%2 $\mathrm{C} 0 \% 5 \mathrm{D}$

Coronado, G. (coord.) (2017). La educación a distancia en México: una década de sostenido esfuerzo institucional. México: Universidad de Guadalajara. Recuperado de http://biblioteca.udgvirtual.udg.mx:8080/jspui/bitstream/123456789/1873/1/Educaci \%C3\%B3n\%20a\%20distancia\%20en\%20M\%C3\%A9xico.pdf

Enfoque estratégico (2019). Características de la educación en línea. Recuperado de https://www.enfoque-estrategico.com/7-caracteristicas-de-la-educacion-en-linea

Espinosa, A. (2017). Profesores "migrantes digitales" enseñando a estudiantes "nativos digitales". MediSur, 15(4), $\quad$ 463-473. $\quad$ Recuperado de http://scielo.sld.cu/scielo.php?script=sci_arttext\&pid=S1727897X2017000400004\&lng=es\&tlng=es

Kearsley, G. (1998). Distance Education Goes Mainstream. T.H.E. Journal, 


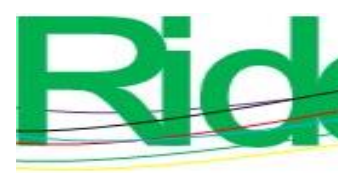

\section{Revista Iberoamericana para la Investigación y el Desarrollo Educativo ISSN $2007-7467$}

25(10). Retrieved from https://www.learntechlib.org/p/86549/

Kok, I., Bester, P. and Esterhuizen, H. (2018). Late Departures from Paper-Based to Supported Networked Learning in South Africa: Lessons Learned. International Journal of Distance Education Technologies (IJDET), 16(1), 56-75. Doi: doi:10.4018/IJDET.2018010104

Licenciaturas en línea (2019). Ranking de las mejores universidades en línea en México. Recuperado de https://estudiarlicenciaturasenlinea.com/ranking-universidades-enlinea-en-mexico/

Moreno, M. (2015). La educación superior a distancia en México. Una propuesta para su análisis histórico. En Zubieta, J. y Rama, C. (eds.), La educación a distancia en México: una nueva realidad universitaria. Recuperado de https://recursos.portaleducoas.org/publicaciones/la-educaci-n-distancia-en-m-xicouna-nueva-realidad-universitaria

Moreno, M. (2017). Presencia de la educación a distancia universitaria en el despertar del siglo XXI. En Coronado, G. (coord.), La educación a distancia en México: una década de sostenido esfuerzo institucional. . México: Universidad de Guadalajara. Recuperado de http://biblioteca.udgvirtual.udg.mx:8080/jspui/bitstream/123456789/1873/1/Educaci \%C3\%B3n\%20a\%20distancia\%20en\%20M\%C3\%A9xico.pdf

Moreno, O. y Cárdenas, M. G. (2012). Educación a distancia: nueva modalidad, nuevos alumnos. Perfiles de alumnos de Psicología en México. Perfiles Educativos, 34(136), 118-136.

Recuperado

de http://www.scielo.org.mx/scielo.php?script=sci_arttext\&pid=S0185$26982012000200008 \& \operatorname{lng}=$ es\&tlng=es

United Nations Educational, Scientific and Cultural Organization [Unesco] (2016). Declaración de París de 2012 sobre los REA. Congreso Mundial sobre los Recursos $\begin{array}{llll}\text { Educativos } & \text { Abiertos } & \text { París. } & \text { Recuperado }\end{array}$ de http://www.unesco.org/new/fileadmin/MULTIMEDIA/HQ/CI/WPFD2009 /Spanish_Declaration.html

United Nations Educational, Scientific and Cultural Organization [Unesco] (2019). Educación superior digital. Recuperado de https://es.unesco.org/themes/educacionsuperior/digital

Zawacki, R. O. and Qayyum, A. (eds.) (2019). Open and Distance Education in Asia, Africa 


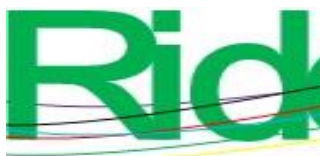

and the Middle East. Doi: https://doi.org/10.1007/978-981-13-5787-9

Zubieta, J. y Rama, C. (coord.) (2015). La educación a distancia en México: una nueva realidad universitaria. México: UNAM. Recuperado de https://recursos.portaleducoas.org/publicaciones/la-educaci-n-distancia-en-m-xicouna-nueva-realidad-universitaria 


\begin{tabular}{|c|c|}
\hline $1=$ & $\begin{array}{l}\text { Revista Iberoamericana para la } \\
\text { Investigación y el Desarrollo Educativo } \\
\text { ISSN } 2007-7467\end{array}$ \\
\hline Rol de Contribución & Autor (es) \\
\hline Conceptualización & Priscila Ortega Gómez \\
\hline Metodología & Priscila Ortega Gómez \\
\hline Software & Priscila Ortega Gómez; Zoe Tamar Infante Jiménez «igual» \\
\hline Validación & Zoe Tamar Infante Jiménez \\
\hline Análisis Formal & Zoe Tamar Infante Jiménez; Priscila Ortega Gómez «igual» \\
\hline Investigación & Priscila Ortega Gómez; Zoe Tamar Infante Jiménez «igual» \\
\hline Recursos & Priscila Ortega Gómez \\
\hline Curación de datos & Zoe Tamar Infante Jiménez \\
\hline $\begin{array}{l}\text { Escritura - Preparación del } \\
\text { borrador original }\end{array}$ & Priscila Ortega Gómez \\
\hline $\begin{array}{l}\text { Escritura - Revisión y } \\
\text { edición }\end{array}$ & Priscila Ortega Gómez; Zoe Tamar Infante Jiménez «igual» \\
\hline Visualización & Zoe Tamar Infante Jiménez \\
\hline Supervisión & Priscila Ortega Gómez \\
\hline Administración de Proyectos & Priscila Ortega Gómez \\
\hline Adquisición de fondos & Priscila Ortega Gómez \\
\hline
\end{tabular}

